\title{
O lazer e o uso de substâncias entre adolescentes: uma revisão integrativa
}

\section{Leisure and substance use among adolescents: a systematic review}

\section{El ocio y uso de substancias entre los adolescentes: uma revisión sistemática}

\section{Diego Eugênio Roquette Godoy Almeida*}

Universidade Federal de São Paulo - Unifesp, São Paulo, São Paulo, Brasil

\section{Denise De Micheli**}

Universidade Federal de São Paulo - Unifesp, São Paulo, São Paulo, Brasil

\section{Andre Luiz Monezi Andrade***}

Pontifícia Universidade Católica de Campinas - PUC-Campinas, Campinas, São Paulo, Brasil

\begin{abstract}
RESUMO
Com base em uma diversidade de definições, existe um consenso sobre o lazer enquanto um fenômeno extraordinário à vida cotidiana. Existem divergências em análises do lazer a partir de perspectivas do tempo, atividade e atitude, correlacionando-o muitas vezes com comportamentos de risco. Objetivamos verificar de forma sistemática se os componentes atitudinais relacionados à experiência do lazer de adolescentes e jovens estão relacionados à redução do uso de substâncias psicoativas. O Método utilizado foi a Revisão Integrativa a partir de consulta às principais bases de dados em saúde, seguida de apresentação dos artigos de acordo com o método PRISMA. Os critérios de revisão e inclusão foram apresentados pela estrutura PICOS. Nos resultados foram recuperados 555 artigos, porém somente 09 artigos preencheram os critérios para análise sistemática dos dados. A conclusão dos estudos sugerem associação da motivação intrínseca e tédio como fatores de risco no lazer para o abuso de substâncias.
\end{abstract}

Palavras-chaves: lazer, adolescentes, transtorno, substâncias.

\begin{abstract}
Introduction: Although there are diversity of concepts, there is a consensus on leisure as an extraordinary phenomenon to everyday life. Studies have divided itself between the prospects for leisure time as activity or attitude, it often correlating with risk behaviors. Objective: the aim of this review is to verify whether attitudinal components related to leisure experience of adolescents and young people are related to the reduction in the use of psychoactive substances. Method: integrative review from the main query databases in health, followed by presentation of the articles according to the
\end{abstract}


PRISMA method. The criteria for review and inclusion were presented by the structure PICOS. Results: we retrieved 555 articles, but only 9 articles met the criteria for systematic analysis of the data. Conclusion: studies suggest intrinsic motivation and boredom as risk factors for substance abuse.

Keywords: leisure, adolescents, disorders, substance.

\section{RESUMEN}

Introducción: Basado en una variedad de definiciones, existe un consenso sobre el ocio mientras un fenómeno extraordinario de la vida cotidiana. Existen diferencias en las análisis de ocio de vez en perspectiva, la actividad y la actitud, lo correlaciona a menudo con un comportamiento de riesgo. Objetivo: Verificar sistemáticamente si los componentes actitudinales relacionados con el ocio de los jóvenes se relacionan con la reducción del uso de sustancias psicoactivas. Método: una revisión integrativa de las consultas a las bases de datos clave de la salud, seguida de la presentación de los artículos de acuerdo con el método PRISMA. Los criterios de inclusión para la revisión y fueron presentados por la estructura PICOS. Resultados: 555 artículos fueron recuperados, pero sólo 09 artículos cumplieron los criterios para el análisis sistemático de los datos. Conclusión: Los estudios han sugerido una asociación de la motivación intrínseca y el aburrimiento como factores de riesgo en el juego para el abuso de sustancias.

Palabras-clave: ocio, adolescentes, transtorno, substancias.

\section{I ntrodução}

O lazer é um relevante objeto de pesquisa multidisciplinar para o qual se voltam antropólogos, comunicólogos, educadores físicos, psicólogos, sociólogos, terapeutas ocupacionais entre outros profissionais. Ao se analisar a relação entre aspectos da saúde e atividades de lazer, Santos, Manfroi, Bertuol, Figueiredo e Marinho (2014) avaliaram a produção latina-americana e observaram uma singnificativa parcela de estudos sobre atividades físicas no "tempo livre", sem utilização de referenciais teóricos específicos, utilizando procedimentos a partir de metodologias quantitativas. Além disso, a perspectiva funcionalista do lazer como "tempo livre", proposta pelo sociólogo francês Joffre Dumazedier é uma das mais utilizadas em estudos brasileiros (Marcellino, 2010). Esse modo de pensar o lazer se fundamenta a partir dos tempos sociais após a revolução industrial em que a concepção de lazer nasce em contraponto às longas jornadas de trabalho, ao esforço pela eficiência e produção acelerada por meio de novas fontes de energia e à alienação da mão-de-obra humana.

De maneira complementar, existe uma abordagem para o lazer em que ele é entendido como atitude. O parâmetro estaria centrado nas experiências de excitação, liberdade, prazer, automotivação (Munusturlar \& Bayrak, 2016). Segundo Dumazedier (2008) o lazer não é algo que se tenha, mas uma experiência que se vive. Neste sentido, o lazer ocorre em qualquer tempo e atividade, e depende 
dos sentimentos e percepções empreendidas neste intento. Abordagens focadas no lazer como um processo de atitude reconhecem que as habilidades e condições socioculturais são elementares, contudo, a ênfase está na possibilidade de negociação com os constrangimentos de toda ordem, na percepção de competências e em diferentes modos de motivação intrínseca (Scott \& Harmon, 2016).

Alguns pesquisadores sugerem que o lazer seja rico em significados e auxilie no desenvolvimento dos adolescentes, por trazer consigo elementos que contribuem para maior autonomia, construção de identidades, competências, iniciativa, atitude cívica, vínculo social e estratégias de adaptação frente a situações estressantes (Powrie, Kolehmainen, Turpin, Ziviani, \& Copley, 2016; Lee, Wu, \& Lin, 2012). Entretanto, em alguns estudos observa-se que esta prática está associada a comportamentos "desviantes", abuso de drogas e comportamentos sexuais de risco (Frade, De Micheli, Andrade, \& Souza-Formigoni, 2013). Por exemplo, Van Havere, Vanderplasschen, Lammertyn, Broekaert e Bellis (2011) investigaram 775 pessoas na Bélgica e observaram que a frequência às casas noturnas e ouvir música eletrônica é fator de risco para o uso de álcool e drogas ilícitas, enquanto que ouvir rock diminui as chances de usar cocaína. Outro estudo em 10 países europeus indicou a preferência pelo dance e do hip-hop como indicador "robusto" para o consumo de substâncias, inclusive o rock (Bogt et al., 2012). Além disso, algumas práticas esportivas parecem estar relacionadas com comportamento de risco ao uso de drogas, em especial esportes coletivos (Bedendo, Andrade, \& Noto, 2015).

O uso de substâncias, portanto, pode estar associado a diversas práticas de lazer, sendo que aproximadamente $65 \%$ dos estudantes brasileiros de ensino fundamental e médio já fizeram uso de álcool na vida de álcool e quase $12 \%$ o fazem de maneira frequente (seis ou mais vezes no último mês) (Carlini, 2005). Conforme os resultados obtidos no $\mathrm{V}$ Levantamento Nacional sobre o Consumo de Drogas Psicotrópicas entre Estudantes, embora tenha-se observado uma redução na prevalência do uso de álcool na vida, houve um aumento progressivo do uso pesado desta substância (vinte dias ou mais no último mês) entre os estudantes. Interessante mencionar que maior consumo foi percebido entre adolescentes de classes sociais mais favorecidas e que o álcool foi a substância com menor média de idade de primeiro uso.

O tabaco é a segunda substância mais consumida entre estudantes brasileiros, com início médio aproximado de 13 anos, sendo que nos adolescentes em situação de rua é a substância com uso mais frequente (Opaleye, Sanchez, Moura, Galduroz, Locatelli \& Noto, 2012). Em relação aos estudos anteriores, observou-se uma redução do consumo de tabaco na maioria das capitais, sendo a região Sul 
com maiores índices. Em relação às substâncias ilícitas, entre 0,6\% a $11,2 \%$ dos jovens já experimentaram maconha com predomínio do sexo masculino (Sanchez, Opaleye, Martins, Ahluwalia, \& Noto, 2010), a despeito das manchetes atuais em torno da cannabis que exageram nas informações negligenciando o fato que a maioria dos estudantes nem sequer provou maconha em algum momento da vida. Estes dados variam de acordo com o tipo de estudo e o público alvo específico.

Em comparação com outros países, o Brasil possui uma das maiores frequências de uso na vida de solventes $(15,4 \%)$, seguido da Grécia e Estados Unidos. Nos adolescentes em situação de rua, existe uma predileção pelos solventes no primeiro uso (Embleton, Mwangi, Vreeman, Ayuku, \& Braitstein, 2013). Outro grupo de substâncias utilizado durante a recreação sãos as drogas estimulantes, sendo que o uso na vida de cocaína foi de $2 \%$ e o de crack $0,7 \%$ (Comis \& Noto, 2012). Os pesquisadores deste estudo ressaltaram que as taxas podem estar subestimadas, uma vez que o método empregado na coleta de dados dirigiu-se a estudantes, excluindo inevitavelmente aqueles que já experimentaram maior rompimento dos vínculos de apoio social como nos casos de evasão escolar. As drogas sintéticas como ecstasy, ketamina e GHB são particularmente usadas por alguns segmentos populacionais, geralmente associadas às raves $\mathrm{e}$ música eletrônica (Palamar, Acosta, Ompad \& Cleland, 2017). Os levantamentos não quantificaram esse tipo de uso.

Tendo em vista a alta prevalência do uso de substâncias psicotrópicas entre adolescentes na população brasileira, o objetivo desta revisão consistiu em verificar de forma sistematizada se a experiência do lazer (como atitude) de adolescentes e jovens estaria associada à redução do uso de substâncias psicotrópicas.

\section{Método}

O presente trabalho configura-se como uma revisão integrativa. Optou-se por esta modalidade de revisão por apresentar uma metodologia mais ampla, permitindo a inclusão estudos com outros desenhos metodológicos além daqueles com ensaios clínicosrandomizados, tais como metodologias qualitativas.

Procedimentos de busca

Definiu-se usar as seguintes bases de dados para a busca dos artigos: LILACS, IBECS e Cochrane-Central interface BVS, ADOLEC, MEDLINE interface PubMed, ERIC e PsyclNFO pela relevância das publicações no campo da saúde. O período investigado foi de 2003 a 2015 sendo que a opção por esta data se deveu a maior quantidade 
de trabalhos encontrados a partir deste período. Foram considerados somente trabalhos em português, inglês ou espanhol. Os descritores utilizados foram consultados nos indexadores do Medical Subject Headings [MESH] ou no [DeCS] e posteriormente combinados por meio dos operadores booleanos AND, OR e AND NOT, conforme ilustrado na Tabela 1. Considerando as possibilidades de combinação, optou-se por explicitar a estratégia usada a fim de possibilitar a replicação dos achados. A Tabela 1 descreve os procedimentos para a recuperação dos manuscritos a partir de diversas bases dedados e os procedimentos de análise e inclusão dos manuscritos para cada uma delas. 
Tabela 1

Descrição dos procedimentos de recuperação de artigos a partir das bases de dados Medline, Eric, Lilacs, Adolec, e PsycINFO e a quantidade de trabalhos recuperados, analisados e incluidos em cada uma delas.

\begin{tabular}{|c|c|c|c|c|}
\hline $\begin{array}{c}\text { Basede } \\
\text { dados }\end{array}$ & Procedimentos de recuperação de artigos & Recuperados & Analisados & Incluidos \\
\hline $\begin{array}{l}\text { Medline } \\
\text { (PubM ed) }\end{array}$ & 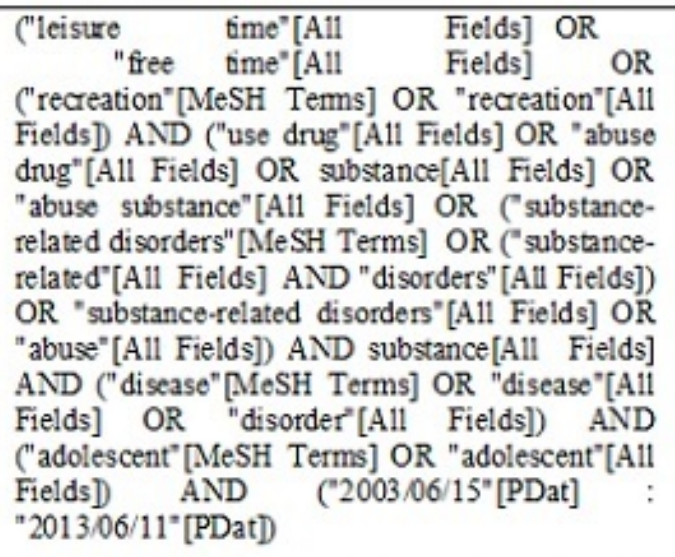 & 267 & 15 & 6 \\
\hline ERIC & $\begin{array}{l}\text { (Keywords: leisure and Keywords: time OR } \\
\text { Keywords: recreation OR Keywords: leisure) and } \\
\text { (Keywords:adolescent) OR (Keywords: "use } \\
\text { substance") OR (Keywords: "abuse } \\
\text { substance disorder") OR (Keywords: "abuse } \\
\text { substance")) and (Publication Type:"Journal } \\
\text { Articles") Publication Date: 2003-2013. }\end{array}$ & 172 & 5 & 3 \\
\hline $\begin{array}{l}\text { LILACS, } \\
\text { IBECS, } \\
\text { COCHRAN } \\
\text { E- } \\
\text { CENTRAL } \\
\text { (BVS) }\end{array}$ & $\begin{array}{l}\text { "atividades de lazer" AND "transtomos } \\
\text { relacionados ao uso de substâncias" AND } \\
\text { adolescente AND db:("IBECS" OR "LILACS" } \\
\text { OR "COCHRANE- CENTRAL") AND } \\
\text { limit:("adolescent") AND la:("en" OR "es" OR } \\
\text { "pt"). }\end{array}$ & 3 & 0 & 0 \\
\hline ADOLEC & $\begin{array}{l}\text { lazer or recreação or ócio [Palavras] and } \\
\text { "transtomo associado ao uso de substâncias" OR } \\
\text { drogas OR "abuso de drogas" }\end{array}$ & 25 & 1 & 0 \\
\hline PsycINFo & $\begin{array}{l}\text { leisure OR recreation OR "free time" AND Any } \\
\text { Field: substance abuse OR drug abuse AND Age } \\
\text { Group: Adolescence AND Year: } 2003 \text { TO } 2013 \\
\text { AND NOT Publication Type: Journal. }\end{array}$ & 88 & 9 & 0 \\
\hline T otal & & 555 & 30 & 9 \\
\hline
\end{tabular}

Critérios de inclusão/exclusão

Após a busca nas bases de dados, realizou-se a análise dos resumos com o objetivo de selecionar somente aqueles pertinentes ao escopo deste estudo. Para tornar mais preciso o processo de seleção dos estudos, utilizou-se a abordagem PICOS (Twa, 2016), um acrômio que faz menção aos cinco componentes representados por cada letra, conforme descrito na Figura 1 . Os critérios de inclusão consideravam os artigos que abordassem a experiência do lazer atitude a partir de metodologias quantitativas e/ou qualitativas. Foram excluídos 
estudos sobre atividades (física, dança, raves, andar de skate, etc), por tratarem o lazer como classe de atividade ou cenário, e também aqueles cujo desfecho estava centrado no uso de anabolizantes, tranquilizantes e analgésicos por exigirem uma discussão mais aprofundada sobre o fenômeno.

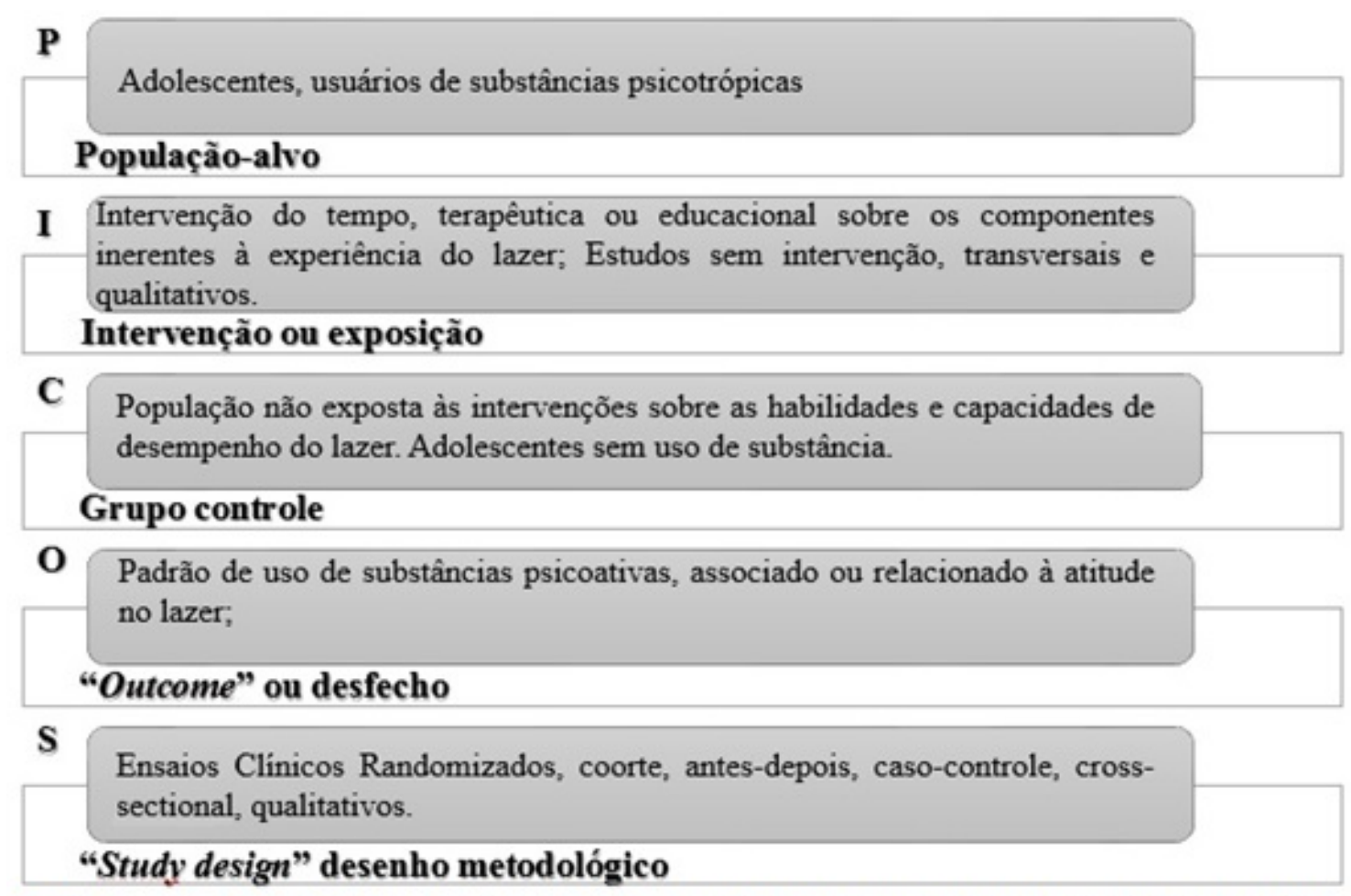

Figura 1. Descrição da metodologia PICOS a partir do acrônimo $\mathbf{P}=$ população alvo, $\mathbf{I}=$ intervenção ou exposição, $\mathbf{C}=$ grupo controle, $\mathbf{O}=$ "outcome" ou desfecho, $\mathbf{S}$ = "study design" desenhos de estudo.

Como não existe um processo normatizado para selecionar e incluir estudos em um processo de revisão de literatura, adotou-se neste estudo a proposta de Sampaio e Mancini (2007) na qual etapa de seleção deve ser feita por pelo menos dois pesquisadores, de forma independente e cega, obedecendo rigorosamente aos critérios de inclusão e exclusão definidos no protocolo de pesquisa. Assim, o método de extração dos dados seguiu os roteiros de análise do Guidelines for critical review form McMaster University Occupational Therapy Evidence-Based Practice Research Group.

Análise de dados

Os procedimentos adotados seguiram o acrônimo PRI SMA (Preferred Reporting I tems for Systematic Reviews and Meta-Analyses), elaborados por um grupo internacional de pesquisadores, cujo objetivo é guiar a apresentação dos dados de uma revisão, de forma sistemática e precisa (Welch et al., 2015). 


\section{Resultados e Discussão}

A pesquisa nas bases Medline, Psycholnfo, Eric, LILACS, IBECS e Cochrane-Central resultou em 555 citações e foram inseridas 2 citações encontradas por busca manual. Destes, 521 trabalhos foram excluídos após análise por pares dos resumos por não se enquadrarem nos critérios do PICOS. Após ajuste dos títulos duplicados, restaram 35 e outros 3 estudos foram descartados porque não estavam disponíveis na íntegra e dois estavam duplicados. Posteriormente, 30 estudos foram lidos integralmente e 24 foram excluídos. Após nova atualização, foram inseridos 3 estudos que se encontravam no prelo mediante o uso da ferramenta do PubMed que envia ao pesquisador um aviso notificando a indexação de um novo manuscrito contendo os descritores pesquisados anteriormente. Todas as etapas foram descritas no fluxo de informações através das diferentes fases (Figura 2). Deste modo, 9 estudos foram incluídos e revisados sistematicamente (Tabela 1).

A partir da busca nas bases de dados, constatou-se a ausência de estudos em português, sendo que os 9 artigos incluídos foram escritos em inglês. Gomes (2004) ao analisar a trajetória histórica dos estudos sobre o lazer no Brasil verifica que é a partir dos anos 80 que a produção científica começa a se avolumar, sob a influência internacional de pensadores como Dumazedier e também pelo esforço de novas Instituições de Ensino Superior e do Serviço Social do Comércio - SESC. Percebe-se que nessa curta trajetória, houve um aumento na produção científica sobre o lazer com enfoque para estudos avaliando identidades de classe, a luta operária, dentro do qual se situava o paradoxo do ócio- negócio.

Nos últimos 30 anos, vários estudos internacionais vêm atentando para a natureza das vulnerabilidades, riscos, oportunidades e o papel que o lazer tem na promoção de bem-estar e desenvolvimento entre os jovens (Weybright, Caldwell, Ram, Smith, \& Wegner, 2015). Nesse sentido, destacam-se a produção de alguns pesquisadores como de Linda Caldwell, Dinna Caffman e Lisa Wegner que investigam adolescentes em vulnerabilidade social na África do Sul. $\mathrm{Na}$ composição total desta revisão, seis estudos provenientes destes pesquisadores foram selecionados por atenderem aos critérios metodológicos. 


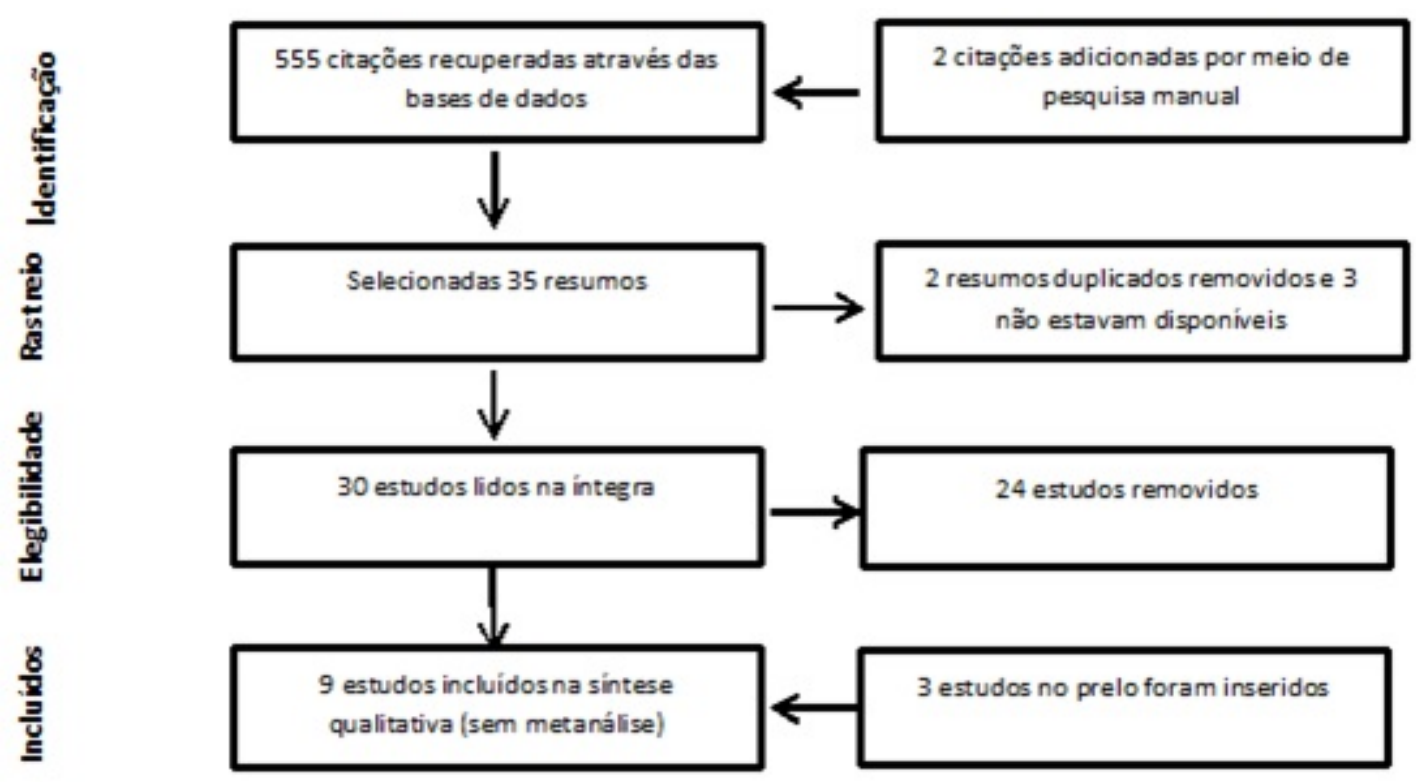

Figura 2. Diagrama do fluxo de seleção e analise dos estudos.

Caldwell, Bradley e Coffman (2009), seguindo a Teoria da Autodeterminação, identificam no programa Time Wise a abordagem para intervir nas habilidades de planejamento do lazer, no desinteresse e no tédio, de tal forma que o adolescente fosse capaz de identificar os próprios interesses e superar os constrangimentos de toda ordem, tendo consequentemente mais chances de envolver-se no chamado "lazer saudável". O sucesso da intervenção viria da abordagem centrada na pessoa, isto é, nas necessidades singulares de cada perfil motivacional. Em seus achados, utilizando a amostra não probabilística de 659 estudantes do sétimo ano de uma zona rural, verificaram que a motivação extrínseca é fator de risco para o uso de tabaco (não apresenta odds ratio). A não randomização da amostra, o uso de instrumentos sem validação e não descrição minuciosa do grupo caso e controle pesaram contra a força de evidência. Além disso, o desenho metodológico não permitiu avaliar a eficácia do programa em termos do tamanho do efeito e o próprio resultado pode ser consequência de possíveis variáveis de confusão. Como resultado, houve associação entre a participação no Time Wise e motivação intrínseca (desfecho primário); o "fumar na vida" foi fator de risco para a motivação e motivação extrínseca, todavia não foi observada uma relação causal ou mesmo efeito da intervenção em relação ao uso de substâncias. Contudo, destaca-se a avaliação dos perfis motivacionais, pressupondo necessidades específicas de intervenção para cada perfil, ao passo que os demais estudos optaram por abordagens uniformes, como se todos pudessem se beneficiar do programa da mesma maneira. 
Tabela 2

Descrição dos estudos incluídos a partir dos objetivos, desenho, amostra, instrumentos utilizados, tipo de intervenção e resultados obtidos.

\begin{tabular}{|c|c|c|c|c|c|c|}
\hline Fonte & Objetivo & $\begin{array}{l}\text { Desenho do } \\
\text { E studo }\end{array}$ & Amostra & Instrumento & Interv enção & Resultado \\
\hline $\begin{array}{l}\text { Eischens et } \\
\text { d., } 2004\end{array}$ & $\begin{array}{l}\text { Verificar se o } \\
\text { DARE PLUS } \\
\text { curriculum está } \\
\text { associado à } \\
\text { redução do uso de } \\
\text { substâncias }\end{array}$ & Caso-controle & $\begin{array}{l}2.241 \\
\text { estudantes } \\
\text { do } 7^{\star x} \text { e } 8^{x} \\
\text { séries }\end{array}$ & $\begin{array}{l}\text { Medida de } \\
\text { frequência de } \\
\text { substâncias e } \\
\text { intenção, perfil } \\
\text { demográfico, } \\
\text { frequência nas } \\
\text { atividades } \\
\text { extracurriculares } \\
\text { e fatores } \\
\text { psicossociais }\end{array}$ & $\begin{array}{l}\text { DARE } \\
\text { PLUS }\end{array}$ & $\begin{array}{l}\text { Diferenças entre } \\
\text { gênero. } \\
\text { Adolescentes que } \\
\text { planejaram e } \\
\text { participaram do } \\
\text { programa } \\
\text { apresentaram } \\
\text { menor vso e } \\
\text { intenção de vso de } \\
\text { álcool }(\mathrm{p}=0.05)\end{array}$ \\
\hline $\begin{array}{l}\text { Rockfellow; } \\
\text { Saules, } \\
2006\end{array}$ & $\begin{array}{l}\text { Investigar } \\
\text { efeito dos fatores } \\
\text { motivacionais na } \\
\text { prática de } \\
\text { esportes e sobre o } \\
\text { vso de } \\
\text { substâncias }\end{array}$ & Caso-controle & $\begin{array}{l}218 \text { jovens } \\
\text { de } 18 \text { a } 24 \\
\text { anos }\end{array}$ & $\begin{array}{l}\text { Backgrawnd } \\
\text { Questiomaire; } \\
\text { Ath atic Activity } \\
\text { Questiomaire }\end{array}$ & & $\begin{array}{l}\text { A motivação } \\
\text { extrinseca teve } \\
\text { efeito significativo } \\
(\mathrm{p}=0.05) \text { sobre o } \\
\text { aumento da } \\
\text { frequência do uso e } \\
\text { quantidade de } \\
\text { álcool, beber em } \\
\text { binge, frequência de } \\
\text { uso de maconha e } \\
\text { tabaco. }\end{array}$ \\
\hline $\begin{array}{l}\text { Lee et al., } \\
2008\end{array}$ & $\begin{array}{l}\text { Verificar a } \\
\text { efetividade da } \\
\text { ação preventiva } \\
\text { dirigida à } \\
\text { comunidade em } \\
\text { relação ao uso de } \\
\text { substâncias }\end{array}$ & Antes-depois & 73 & $\begin{array}{l}\text { Entrevistas semi- } \\
\text { estruturadas. }\end{array}$ & $\begin{array}{l}\text { Atividades } \\
\text { recreativas } \\
\text { voltadas para } \\
\text { relações com } \\
\text { a } \\
\text { comvnidade }\end{array}$ & $\begin{array}{l}\text { Redução } \\
\text { significativa do } \\
\text { consumo de } \\
\text { maconha no sexo } \\
\text { feminino, = } 16 \text { ano }\end{array}$ \\
\hline $\begin{array}{l}\text { Caldwell et } \\
\text { d, } 2009\end{array}$ & $\begin{array}{l}\text { Verificar se o vso } \\
\text { de substâncias é } \\
\text { fator associado ao } \\
\text { perfil } \\
\text { motivacional }\end{array}$ & $\begin{array}{l}\text { Cross- } \\
\text { sectional, } \\
\text { regressão. }\end{array}$ & $\begin{array}{l}617 \\
\text { estudantes } \\
\text { da } 7 \text { 'série de } \\
\text { zona rural) }\end{array}$ & $\begin{array}{l}\text { Questionário sem } \\
\text { validação (perfil } \\
\text { motivacional e } \\
\text { uso de } \\
\text { tabaco/álcool) }\end{array}$ & - & $\begin{array}{l}\text { O perfil } \\
\text { motivacional não } \\
\text { teve efeito } \\
\text { significativo } \\
\text { ( } \mathrm{p}=.0 .05) \text { sobre o } \\
\text { vso, porém já ter } \\
\text { fumado é fator } \\
\text { associado } \\
\text { motivação } \\
\text { motivação } \\
\text { extrinseca. }\end{array}$ \\
\hline $\begin{array}{l}\text { Sharp et al., } \\
2011\end{array}$ & $\begin{array}{l}\text { Examinar } \\
\text { longitudinal } \\
\text { associação entre } \\
\text { o lazer boredom, } \\
\text { novos interesses } \\
\text { no lazer, lazer } \\
\text { saudável e uso de } \\
\text { substâncias }\end{array}$ & Coorte & $\begin{array}{l}1.118 \\
\text { Média = } 13 \\
\text { anos }\end{array}$ & $\begin{array}{l}\text { Construido a } \\
\text { partir de sub- } \\
\text { escalas do Leiswre } \\
\text { Experience } \\
\text { Battery for } \\
\text { adolescentes e } \\
\text { cutros itens do } \\
\text { tipolikert }\end{array}$ & - & $\begin{array}{l}\text { O lazer boredom } \\
\text { estava positivamente } \\
\text { associado ao uso de } \\
\text { substâncias (?36\% as } \\
\text { chances para cada } \\
\text { unidade). Lazer } \\
\text { saudável associado } \\
\text { negativamente com } \\
\text { vso de álcool e } \\
\text { maconha. Interesses } \\
\text { no lazer associado } \\
\text { negativamente com } \\
\text { vsar maconha. }\end{array}$ \\
\hline
\end{tabular}




\begin{tabular}{|c|c|c|c|c|c|}
\hline $\begin{array}{l}\text { Tibbits et } \\
\text { d. } 2011\end{array}$ & $\begin{array}{l}\text { Avaliar a eficácia } \\
\text { do programa } \\
\text { Health Wise na } \\
\text { prevenção ao uso } \\
\text { de miltiplas } \\
\text { drogas e } \\
\text { comportamento } \\
\text { sexual de risco. }\end{array}$ & $\begin{array}{l}4.040 \\
(15 \mathrm{a} 1 \\
\text { ancs) }\end{array}$ & $\begin{array}{l}\text { Questi } \\
\text { sobre } \\
\text { de } \\
\text { substâs } \\
\text { prática } \\
\text { uso } \\
\text { preser }\end{array}$ & $\begin{array}{l}\text { nário } \\
\text { Frequência Health } W \text { ise } \\
\text { so de } \\
\text { ias, } \\
\text { sexual e } \\
\text { de } \\
\text { tivo. }\end{array}$ & 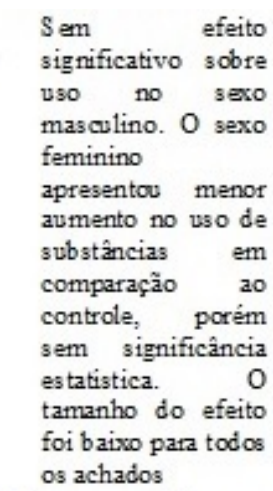 \\
\hline $\begin{array}{l}\text { Wegner, } \\
2011\end{array}$ & $\begin{array}{l}\text { Investigar a } \\
\text { percepcão de } \\
\text { adolescentes sobre o } \\
\text { Leisure Boredom e } \\
\text { comportamentos de } \\
\text { risco no tempo livre. }\end{array}$ & $\begin{array}{l}\text { Estudo qualitativo. } \\
\text { Amostra de } \\
\text { conveniência com } 3 \\
\text { grupos: estudantes, } \\
\text { adolescentes } \\
\text { evadidos da escola; } \\
\text { adolescentes da } \\
\text { comvnidade de Cape } \\
\text { Town }\end{array}$ & $\begin{array}{l}32 \\
\text { adolescentes } \\
\text { entre } 13 \text { e } 20 \\
\text { anos }\end{array}$ & $\begin{array}{l}\text { Grupo Focal; } \\
\text { Fotos tiradas } \\
\text { pelos } \\
\text { adolescentes } \\
\text { durante do tempo } \\
\text { livre. }\end{array}$ & $\begin{array}{l}\text { Os participantes } \\
\text { sentiram que o tédio } \\
\text { foi } \\
\text { parte da vida, levando- } \\
\text { os a comportamentos } \\
\text { de risco. Fica evidente } \\
\text { o papel das privaçóes } \\
\text { socioeconômicas. }\end{array}$ \\
\hline $\begin{array}{l}\text { Weybright } \\
\text { et } d, 2014\end{array}$ & $\begin{array}{l}\text { Compreender como o } \\
\text { lazer saudável } \\
\text { contribui para o uso } \\
\text { de substâncias }\end{array}$ & Coorte & $\begin{array}{l}5.799 \\
\text { estudantes } \\
(12 \text { a } 18 \\
\text { anos) }\end{array}$ & $\begin{array}{l}\text { Heathy L sisure } \\
\text { Perceived } \\
\text { Parental Over- } \\
\text { Control } \\
\text { Frequência de } \\
\text { Uso de } \\
\text { substâncias }\end{array}$ & $\begin{array}{l}\text { Houve associação } \\
\text { significativa entre uso } \\
\text { de substâncias e o } \\
\text { "Lazer S avdável". }\end{array}$ \\
\hline $\begin{array}{l}\text { Weybright } \\
\text { et } d \text {., } 2015\end{array}$ & $\begin{array}{l}\text { Compreender a } \\
\text { associação entre ouso } \\
\text { de substâncias e o } \\
\text { tédio nolazer }\end{array}$ & Coorte & $\begin{array}{l}2.580 \\
\text { estudantes } \\
(12 \text { a } 19 \\
\text { anos) }\end{array}$ & $\begin{array}{l}\text { Leiswe Baredom, } \\
\text { adaptado do } \\
\text { Leiswe } \\
\text { Experience } \\
\text { Battery e registro } \\
\text { da frequência de } \\
\text { uso de cada } \\
\text { substância. }\end{array}$ & $\begin{array}{l}\text { Quanto maior o nivel } \\
\text { de tédio no lazer, mais } \\
\text { os adolescentes estão } \\
\text { propensos a usarem } \\
\text { substâncias. }\end{array}$ \\
\hline
\end{tabular}

Em relação à motivação extrínseca, Rockafellow e Saules (2006) detectaram efeito significante na população de esportistas e atletas. A pesquisa foi realizada com estudantes de psicologia, dentro da faixa etária de 18 a 24 anos, selecionados por conveniência. Atletas com motivação extrínseca apresentaram maior frequência de consumo de bebidas alcoólicas, de maconha e tabaco. Eles usavam em maior quantidade, inclusive o beber pesado episódico (binge). É possível que a coleta de dados da frequência e quantidade de uso de substâncias, por meio de instrumento validado permitiria apreciação mais precisa do efeito da motivação externa sobre o uso de álcool, tabaco e maconha. É interessante ressaltar que existem diversos estudos avaliando o efeito das práticas esportivas no uso de substâncias e os resultados ainda são contraditórios (Kingsland et al., 2015).

O Leisure Boredom ou Tédio no Lazer parece ser um dos achados mais em relação ao uso de substâncias entre adolescentes, ainda que não esteja claro como funciona esta relação (Weybright et al., 2015). O tédio é um fenômeno complexo, descrito como ansiedade, perda de significado e propósito nas atividades, acompanhado por sentimento 
de insatisfação, irritabilidade, estresse e sensação de aprisionamento (Crockett, Myhre, \& Rokke, 2015). Assim, a propensão ao tédio tem sido associada a alguns tipos de transtornos de humor, raiva e agressividade, menor satisfação com o trabalho e com a vida, maior busca de sensações e baixos níveis de autonomia (Spaeth, Weichold, \& Silbereisen, 2015). Deste modo, o Leisure Boredom ocorreria quando os indivíduos percebem que suas experiências são insuficientes para lhes proporcionarem excitação e satisfação (Miller, Caldwell, Weybright, Smith, Vergnani, \& Wegner, 2014).

Wegner (2011) investigou a percepção de tédio no lazer e os comportamentos de risco de adolescentes sul-africanos durante 0 tempo livre a partir de um desenho metodológico qualitativo. A amostra foi composta por três grupos de adolescentes (entre 13 e 20 anos), sendo um grupo da comunidade, outro de estudantes e um terceiro com adolescentes evadidos dessa mesma escola. Os participantes relataram suas experiências no tempo livre tirando fotografias, que serviram de base para discussões em grupos focais. Os resultados indicaram que os adolescentes ficavam entediados em seu tempo livre, principalmente porque não tinham nada para fazer. A baixa condição socioeconômica levava à privação ocupacional e desequilíbrio no tempo livre, o que alimentava a sensação de tédio, a despeito da oportunidade de socialização nas esquinas e ruas. Neste sentido, Whittemore e Knafl (2005) procuraram compreender a associação entre o uso de substâncias entre adolescentes e o tédio, diferenciando aqueles que pareciam tê-lo como "traço" de personalidade e aqueles que pudessem passar por "estados" entediantes e concluíram que o uso de substâncias estava associado a ambos os casos. Contudo, o uso oscilou mais para aqueles "entediados crônicos".

Já no estudo de Sharp et al. (2011), analisando o corte de 1.118 estudantes oriundos da África do Sul, observaram que o tédio no lazer ("leisure boredom"), lazer saudável e interesses no lazer não tiveram mudanças significativas a partir do aumento da idade dos adolescentes, mas eram fatores associados ao uso de substâncias, com tamanho de efeito entre fraco a médio quanto a linha de base e durante o follow-up ( 7 ondas). Deste modo, o uso de substâncias foi fator preditivo para mudanças nas experiências de lazer. Quanto ao impacto dos programas voltados ao lazer na prevenção ao uso de substâncias, ainda não se existe um consenso em relação aos achados, pois existem muitas formas de intervenções e procedimentos diversos.

No programa Health Wise e a hipótese dos autores é que a intervenção atuaria como fator protetivo do abuso de substâncias e comportamentos sexuais de risco (Tibbits et al., 2011). A populaçãoalvo era composta por estudantes da África do Sul, do $8^{\circ}$ ao 10 ㅇ grau, avaliados longitudinalmente por cinco vezes em coortes, 
sempre no começo e final do ano letivo. Ao longo deste tempo ocorreu um drop-out de $50 \%$, fato não justificado no estudo. Os resultados sugerem impacto positivo do programa Heath Wise no sexo feminino, tendo menor aumento de uso de substâncias quando comparado ao grupo controle, mas sem significância estatística. O gênero tem sido importante variável a ser considerada ao se avaliar o impacto de programas como este. Já no estudo de Weybright et al. (2014), os autores acumularam ao longo dos cinco anos de implantação do Health Wise dados capazes de avaliar uma possível associação entre o chamado "Lazer Saudável" e o uso de substâncias. Com efeito, fica demonstrada a associação, mas sem um detalhamento longitudinal dos dados apresentados. Assim como em outros estudos, os instrumentos não foram apresentados em suas qualidades psicométricas. A partir de um olhar crítico sobre a tendência disciplinadora impressa na perspectiva teórica da "Problem Behavior Theory" pela qual o risco é tomado enquanto "tendência ao comportamento não convencional" e o saudável, como sinônimo de normatividade.

Tanto no programa Health Wise (Tibbits, Smith, Caldwell, \& Flisher, 2011) quanto no Programa Dirigido à Comunidade (Lee, Conigrave, Clough, Wallace, Silins \& Rawles, 2008), as capacidades para o engajamento no lazer foram trabalhadas de forma geral, sem análise prévia dos constrangimentos e necessidades de cada componente. A intervenção proposta, portanto, pode estar distante dos fatores relacionados ao lazer capazes de evitar o uso abusivo de substâncias. O DARE PLUS Curriculum é descrito como abordagem de 10 sessões focadas em nas habilidades de resistência ao uso de sustâncias, negociação de conflitos e cidadania, envolvendo líderes e pais de modo que o estudante possa ser auxiliado na capacidade de planejar suas atividades extracurriculares (Secades-Villa \& FernándezHermida, 2003). Neste trabalho, 24 escolas sofrem a intervenção dirigida para estudantes do 70 e 8ㅇ ano e aqueles estudantes que planejaram e participaram deste programa apresentaram menor intenção e uso de substâncias além de maior envolvimento nas atividades extracurriculares. Estes achados corroboram a importância da autodeterminação em programas preventivos dirigidos aos adolescentes, principalmente em relação à adesão. Na análise por gênero, adolescentes do sexo masculino reduziram significativamente o consumo e a intenção de beber em relação às mulheres. Contudo, ao descrever os procedimentos no corpo do artigo, não ficou claro se - lazer foi utilizado nas atividades extracurriculares somente como reforçador da presença no programa, o que impossibilitou a análise de possíveis ganhos advindos do desenvolvimento e de capacidades para o lazer.

Já Lee et al. (2008) avaliaram uma intervenção comunitária com comunidades indígenas da Austrália. Os resultados foram eficazes na 
redução do consumo de maconha pela população pertencente à tribo investigada, porém a ausência de um grupo controle e descrição minuciosa da amostra impossibilitaram afirmar se os benefícios aferidos se ocorreram somente a partir da intervenção. Todos os estudos descritos neste artigo alertam sobre possíveis vieses surgidos a partir do uso de instrumentos autoaplicáveis ou entrevista para identificação do uso de substâncias. Embora se saiba da tendência à omissão ou respostas próximas ao desejável e não ao real, estudiosos tem reafirmado a validade de tais métodos para esses achados.

O presente estudo possui limitações que devem ser levadas em consideração. É possível que existam outros estudos que não foram considerados nesta revisão por não serem escritos em inglês, português e/ou espanhol. Além disso, a relação entre atividades de lazer e o uso de drogas foi avaliada a partir de um recorte, com uma amostra composta somente por adolescentes. Como este é um fenômeno muito amplo, pois envolve uma série de variáveis, é possível que esta relação se estabeleça de outra maneira quando avaliada uma amostra por adultos.

\section{Conclusão}

O presente trabalho verificou a relação entre os componentes subjetivos do lazer o uso de substâncias em adolescentes, e foram encontradas uma quantidade reduzida de publicações. Embora seja apontado o crescente uso de substâncias em todo o mundo e de maneira mais precoce, constata-se a escassez de estudos capazes de captar o fenômeno do lazer em sua dimensão atitudinal. Estes trabalhos favorecem o desenvolvimento de novas pesquisas sobre programas preventivos, nos quais destaca-se a importância da motivação intrínseca no lazer como fator de proteção ao uso de substâncias, segundo a Teoria da Autodeterminação.

Nesta revisão, não foram detectados ensaios clínicos randomizados que são considerados como padrão-ouro na pirâmide de evidências para avaliar a eficácia de intervenções. Ainda assim, estudos avaliados nesta revisão corroboram a hipótese de que o investimento nos componentes intrínsecos ao lazer traria possíveis desfechos benéficos aos adolescentes que fazem abuso de substâncias.

Por fim, ressalta-se a importância desta síntese de evidências a fim de fomentar o interesse de educadores, profissionais da saúde, cultura e política quanto à potencialização do lazer em adolescentes na prevenção ao uso de drogas. Como novas possibilidades de estudo a partir deste trabalho, existe a possibilidade da avaliação da associação entre o lazer e o uso de substâncias em amostras estratificadas (adultos e idosos, por exemplo) permitindo uma análise 
comparativa com outros trabalhos e uma discussão mais aprofundada dos achados a partir de elementos condicionais do lazer como classe social, gênero/sexo, raça/cor e outros marcadores sociais.

\section{Referências}

Bedendo, A., Andrade, A. L. M., \& Noto, A. R. (2015). Prática esportiva e uso de substâncias entre estudantes do ensino médio: diferentes perspectivas dessa relação. SMAD. Revista Eletrônica Saúde Mental Álcool e Drogas, 11(2), 85-96. Doi: 10.11606/issn. 1806-6976.v11i2p85-96

Bogt, T. F., Gabhainn, S. N., Simons-Morton, B. G., Ferreira, M., Hublet, A. \& Godeau, E., et al. (2012). Dance is the new metal: adolescent music preferences and substance use across Europe. Substance Use \& Misuse, 47(2), 130-142. Doi: 10.3109/10826084.2012.637438

Caldwell, L. L., Bradley, S., \& Coffman, D. (2009). A person-centered approach to individualizing a school-based universal preventive intervention. The American Journal of Drug and Alcohol Abuse, 35(4), 214-219. Doi: 10.3109/10826084.2012.637438

Carlini, E. A. (2005). V Levantamento Nacional sobre o consumo de drogas psicotrópicas entre estudantes do ensino fundamental e médio da rede pública de ensino nas 27 capitais brasileiras: 2004 V Levantamento Nacional sobre o Consumo de Drogas Psicotrópicas entre Estudantes do Ensino Fundamental e Médio da Rede Pública de Ensino nas 27 Capitais Brasileiras: 2004: UNIFESP Centro Brasileiro de informações sobre Drogas Psicotrópicas. Recuperado de: http://www2.unifesp.br/dpsicobio/cebrid/levantamento_brasil2/ 000-Iniciais.pdf

Comis, M. A., \& Noto, A. R. (2012). Reasons for not using ecstasy: a qualitative study of non-users, ex-light users and ex-moderate users. BMC Public Health, 12, 353. Doi: 10.1186/1471-245812-353

Crockett, A. C., Myhre, S. K., \& Rokke, P. D. (2015). Boredom proneness and emotion regulation predict emotional eating. Journal of Health Psychology, 20(5), 670-680. Doi: $10.1177 / 1359105315573439$

Dumazedier, J. (2008). Sociologia Empírica do Lazer (Edição Ed.). São Paulo: Perspectiva, SESC.

Eischens, A., Komro, K. A., Perry, C. L., Bosma, L. M., \& Farbakhsh, K. (2004). The association of extracurricular activity participation with substance use among youth in the DARE Plus Project. American Journal of Health Education, 35(2), 68-75. Doi: 10.1080/19325037.2004.10603612 
Embleton, L., Mwangi, A., Vreeman, R., Ayuku, D., \& Braitstein, P. (2013). The epidemiology of substance use among street children in resource-constrained settings: a systematic review and meta-analysis. Addiction, 108(10), 1722-1733. Doi: 10.1111 /add. 12252

Frade, I. F., De Micheli, D., Andrade, A. L.M, \& Souza-Formigoni, M. L. (2013). Relationship between stress symptoms and drug use among secondary students. The Spanish Journal of Psychology, 16, E4. Doi: 10.1017/sjp.2013.5

Gomes, C. M. (2004). Pesquisa científica em lazer no Brasil: bases documentais e teóricas. Dissertação de mestrado nãopublicada. Universidade Federal do Rio Grande do Sul, Porto Alegre, Brasil. Retirado de: http://www.ufrgs.br/infotec/teses03-04/resumo_2987.html

Kingsland, M., Wolfenden, L., Tindall, J., Rowland, B. C., Lecathelinais, C., Gillham, K. E., Wiggers, J. H. (2015). Tackling risky alcohol consumption in sport: a cluster randomised controlled trial of an alcohol management intervention with community football clubs. Journal of Epidemiology and Community Health, 69(10), 993-999. Doi: 10.1136/jech-2014204984

Lee, Cheng-Jong, Wu, Chao-Sen, \& Lin, Chiung-Tzu. (2012). Leisure activity and coping with stress: adolescents as case study. Quality \& Quantity, 46(3), 979-991. Doi: 10.1007/s11135-0129692-5

Lee, K. S., Conigrave, K. M., Clough, A. R., Wallace, C., Silins, E., \& Rawles, J. (2008). Evaluation of a community-driven preventive youth initiative in Arnhem Land, Northern Territory, Australia. Drug and Alcohol Review, 27(1), 75-82. Doi: 10.1080/09595230701711124

Marcellino, N. C. (2010). Contribuições de autores clássicos modernos e contemporâneos para os estudos do lazer. Licere (Online), 13(4). Retirado

https://seer.ufmg.br/index.php/licere/article/view/521

Miller, J. A., Caldwell, L. L., Weybright, E. H., Smith, E. A., Vergnani, T., \& Wegner, L. (2014). Was Bob Seger Right? Relation Between Boredom in Leisure and [Risky] Sex. Leisure Science, 36(1), 52-67. Doi: 10.1080/01490400.2014.860789

Munusturlar, S., \& Bayrak, C. (2016). Developing the Leisure Education Scale. World Leisure Journal, 1-20. Doi: 10.1080/16078055.2016.1251489

Opaleye, E. S., Sanchez, Z. M., Moura, Y. G., Galduroz, J. C., Locatelli, D. P., \& Noto, A. R. (2012). The Brazilian smoker: a survey in the largest cities of Brazil. Revista Brasileira de Psiquiatria, 34(1), 43-51. Doi: 10.1590/S151644462012000100009 
Palamar, J. J., Acosta, P., Ompad, D. C., \& Cleland, C. M. (2017). Self-reported ecstasy/MDMA/"Molly" use in a sample of nightclub and dance festival attendees in New York City. Substance Use \& Misuse, 52(1), 82-91. Doi: 10.1080/10826084.2016.1219373

Powrie, B., Kolehmainen, N., Turpin, M., Ziviani, J., \& Copley, J. (2015). The meaning of leisure for children and young people with physical disabilities: a systematic evidence synthesis. Developmental Medicine \& Child Neurology, 57(11), 993-1010. Doi: $10.1111 /$ dmcn. 12788

Rockafellow, B. D., \& Saules, K. K. (2006). Substance use by college students: the role of intrinsic versus extrinsic motivation for athletic involvement. Psychology of Addictive Behaviors, 20(3), 279-287. Doi: 10.1037/0893-164X.20.3.279

Sampaio, R. F., \& Mancini, M. C. (2007). Estudos de revisão sistemática: um guia para síntese criteriosa da evidência científica. Brazilian Journal of Physical Therapy, 11(1), 83-89. Doi: 10.1590/S1413-35552007000100013

Sanchez, Z. M., Opaleye, E. S., Martins, S. S., Ahluwalia, J. S., \& Noto, A. R. (2010). Adolescent gender differences in the determinants of tobacco smoking: a cross sectional survey among high school students in Sao Paulo. BMC Public Health, 10, 748. Doi: 10.1186/1471-2458-10-748

Santos, P. M., Manfroi, M. N., Bertuol, C., Figueiredo,P. J., \& Marinho, A. (2014). Abordagens sobre o lazer na literatura latinoamericana em ciências da saúde: uma revisão na base de dados lilacs. Revista Brasileira de Estudos do Lazer, 1(2), 117139.

Retirado

de: https://seer.ufmg.br/index.php/rbel/article/view/780

Scott, D., \& Harmon, J. (2016). Extended Leisure Experiences: A Sociological Conceptualization. Leisure Sciences, 38(5), 482488. Doi: 10.1080/01490400.2015.1123126

Secades-Villa, R., \& Fernández-Hermida, J. R. (2003). The validity of self-reports in a follow-up study with drug addicts. Addictive behaviors, 28(6), 1175-1182. Doi: 10.1016/S03064603(02)00219-8

Sharp, E. H., Coffman, D. L., Caldwell, L. L., Smith, E. A., Wegner, L., Vergnani, T., \& Mathews, C. (2011). Predicting substance use behavior among South African adolescents: The role of leisure experiences across time. International Journal of Behavioral Development, 35(4), 343-351. Doi: $10.1177 / 0165025411404494$

Spaeth, M., Weichold, K., \& Silbereisen, R. K. (2015). The development of leisure boredom in early adolescence: Predictors and longitudinal associations with delinquency and 
depression. Developmental Psychology, 51(10), 1380-1394. Doi: $10.1037 / \mathrm{a} 0039480$

Tibbits, M. K., Smith, E. A., Caldwell, L. L., \& Flisher, A. J. (2011). Impact of HealthWise South Africa on polydrug use and highrisk sexual behavior. Health Education Research, 26(4), 653663. Doi: 10.1093/her/cyr024

Twa, M. D. (2016). Evidence-Based Clinical Practice: Asking Focused Questions (PICO). Optometry \& Vision Science, 93(10), 11871188. Doi: 10.1097/OPX.0000000000001006

Van Havere, T., Vanderplasschen, W., Lammertyn, J., Broekaert, E., $\&$ Bellis, M. (2011). Drug use and nightlife: more than just dance music. Substance Abuse Treatment, Prevention, and Policy, 6, 18-29. Doi: 10.1186/1747-597X-6-18

Wegner, L. (2011). Through the lens of a peer: Understanding leisure boredom and risk behaviour in adolescence. South African Journal of Occupational Therapy 41(1), 18-24. Retirado de: http://www. sajot.co.za/index.php/sajot/article/download/15/21

Welch, V., Petticrew, M., Petkovic, J., Moher, D., Waters, E., White, H., \& Tugwell, P. (2015). Extending the PRISMA statement to equity-focused systematic reviews (PRISMA-E 2012): explanation and elaboration. International Journal for Equity in Health, 14(1), 92. Doi: 10.1186/s12939-015-0219-2

Weybright, E. H., Caldwell, L. L., Ram, N., Smith, E., \& Jacobs, J. (2014). The Dynamic Association between Healthy Leisure and Substance Use in South African Adolescents: A State and Trait Perspective. World Leisure Journal, 56(2), 99-109. Doi: 10.1080/16078055.2014.903726

Weybright, E. H., Caldwell, L. L., Ram, N., Smith, E. A., \& Wegner, L. (2015). Boredom Prone or Nothing to Do? Distinguishing Between State and Trait Leisure Boredom and its Association with Substance Use in South African Adolescents. Leisure Sciences, 37(4), 311-331. Doi: $10.1080 / 01490400.2015 .1014530$

Whittemore, R., \& Knafl, K. (2005). The integrative review: updated methodology. Journal of advanced nursing, 52(5), 546-553. Doi: $10.1111 / j .1365-2648.2005 .03621 . x$

\footnotetext{
Endereço para correspondencia Diego Eugênio Roquette Godoy Almeida Universidade Federal de São Paulo - Unifesp Rua Napoleão de Barros, 1038, Vila Clementino, CEP 04024-003, São Paulo - SP, Brasil

Endereço eletrônico: diego.godoy@yahoo.com.br
} 


\section{Denise De Micheli}

Universidade Federal de São Paulo - Unifesp

Rua Napoleão de Barros, 1038, Vila Clementino, CEP 04024-003, São Paulo - SP, Brasil

Endereço eletrônico: demicheli_unifesp@gmail.com

\section{Andre Luiz Monezi Andrade}

Pontifícia Universidade Católica de Campinas - PUC-Campinas

Av. John Boyd Dunlop, S/N, Jardim Londres, CEP 13034-685, Campinas - SP, Brasil

Endereço eletrônico: andre.andrade@puc-campinas.edu.br

Recebido em: 15/02/2016

Reformulado em: 10/03/2017

Aceito em: 24/03/2017

\section{Notas}

* Bacharel em Terapia Ocupacional, Mestre e Doutorando em Educação e Saúde na Infância e Adolescência da Universidade Federal de São Paulo.

** Graduada em Psicologia. Mestre, Doutora e Pós-Doutora em Psicobiologia pela Universidade Federal de São Paulo. Professora Adjunta da Universidade Federal de São Paulo.

*** Graduado em Psicologia. Mestre, Doutor e Pós-Doutor em Psicobiologia pela Universidade Federal de São Paulo. Docente Pesquisador da PUC-Campinas.

Este artigo de revista Estudos e Pesquisas em Psicologia é licenciado sob uma Licença Creative Commons Atribuição-Não Comercial 3.0 Não Adaptada. 